\title{
Measurement of quarkonia production in heavy-ion collisions with the ATLAS detector
}

\author{
Jakub Andrzej Kremer* on behalf of the ATLAS Collaboration \\ AGH University of Science and Technology, Kraków, Poland \\ E-mail: jakub.kremerecern.ch
}

\begin{abstract}
The suppression of heavy quarkonia states in heavy-ion collisions is a phenomenon understood as a consequence of QGP formation in the hot, dense system formed in nucleus-nucleus collisions at the LHC. In addition to hot matter effects in heavy-ion collisions, cold nuclear effects may also affect quarkonia production. Therefore, a full assessment requires detailed studies on the effects present in both $\mathrm{A}+\mathrm{A}$ and $p+\mathrm{A}$ collisions. Based on $p+\mathrm{Pb}$ data collected in 2013 and $p p$ and $\mathrm{Pb}+\mathrm{Pb}$ data collected in 2015 at the LHC, the ATLAS experiment has studied prompt and non-prompt $J / \psi$ and $\psi(2 \mathrm{~S})$ productions as well as $\Upsilon(n \mathrm{~S})$ production via the di-muon decay final states. The results of the various measurements are discussed.
\end{abstract}

The European Physical Society Conference on High Energy Physics 5-12 July 2017

Venice, Italy

\footnotetext{
* Speaker.
} 


\section{Introduction}

Ultrarelativistic heavy-ion collisions may lead to the appearance of a strongly interacting quark-gluon plasma (QGP), a state of matter which consists of deconfined quarks and gluons. The idea that quarkonia - bound states of a quark and antiquark - could be used to probe this hot and dense medium was first proposed by Matsui and Satz [1]. Quarkonia are usually produced in the early stages of a collision - before the QGP is formed - and are sensitive to properties of the medium through several mechanisms: suppression of production due to screening by free colour charges in the plasma, statistical recombination of $q \bar{q}$ pairs leading to regeneration and $b$-quark energy loss in the medium. However, these QGP effects present in nucleus-nucleus collisions are entangled with cold nuclear matter (CNM) effects, which can be studied directly with protonnucleus collisions, where the creation of QGP is not expected. These CNM effects include: nuclear modifications to parton distribution functions, initial parton energy loss and nuclear absorption of the bound $q \bar{q}$ state.

The modification of quarkonia production in proton-lead $(p+\mathrm{Pb})$ collisions relative to protonproton $(p p)$ collisions at the same energy is quantified with the nuclear modification factor defined as:

$$
R_{p \mathrm{~Pb}}=\frac{1}{A_{\mathrm{Pb}}} \frac{\sigma_{p+\mathrm{Pb}}}{\sigma_{p p}}
$$

where $\sigma_{p+\mathrm{Pb}}$ and $\sigma_{p p}$ are production cross-sections of a given quarkonium state measured in $p+\mathrm{Pb}$ and $p p$ collisions, respectively, while $A_{\mathrm{Pb}}$ is the mass number of the lead nucleus (208). For leadlead $(\mathrm{Pb}+\mathrm{Pb})$ collisions the nuclear modification factor is defined taking into account the collision geometry:

$$
R_{\mathrm{AA}}=\frac{N_{\mathrm{AA}}}{\left\langle T_{\mathrm{AA}}\right\rangle \times \sigma_{p p}},
$$

where $N_{\mathrm{AA}}$ is the per-event quarkonium production yield measured in $\mathrm{Pb}+\mathrm{Pb}$ collisions, $\left\langle T_{\mathrm{AA}}\right\rangle$ is the average nuclear thickness function and $\sigma_{p p}$ is the quarkonium production cross-section measured in $p p$ collisions.

This report presents ATLAS [2] measurements of charmonia production in $p p, p+\mathrm{Pb}$ and $\mathrm{Pb}+\mathrm{Pb}$ collisions at $\sqrt{s_{\mathrm{NN}}}=5.02 \mathrm{TeV}[3,4]$ and measurements of bottomonia production in $p p$ and $p+\mathrm{Pb}$ collisions at $\sqrt{s_{\mathrm{NN}}}=5.02 \mathrm{TeV}[3]$.

\section{Charmonia production in $p p, p+\mathrm{Pb}$ and $\mathrm{Pb}+\mathrm{Pb}$ collisions}

Measurements of quarkonia production are performed by ATLAS in the di-muon decay channels and thus use events collected with triggers requiring at least two muons to be reconstructed. Candidate events for charmonia production are formed from opposite-sign di-muon pairs by requiring the two muons to come from a common decay vertex. The pairs are also required to have an invariant mass $m_{\mu \mu}$ between 2.6 and $4.2 \mathrm{GeV}$. In $p+\mathrm{Pb}$ collisions, the selected events are categorised in bins of di-muon transverse momentum $p_{\mathrm{T}}$ and centre-of-mass rapidity $y^{*}$ in the ranges: $8<p_{\mathrm{T}}<40 \mathrm{GeV},-2<y^{*}<1.5$; in $\mathrm{Pb}+\mathrm{Pb}$ collisions the ranges are: $9<p_{\mathrm{T}}<40 \mathrm{GeV},|y|<2$. 
In order to extract charmonia yields in each kinematic bin, an unbinned maximum-likelihood fit is performed simultaneously in $m_{\mu \mu}$ and the pseudo-proper lifetime $\tau_{\mu \mu}=L_{x y} m_{\mu \mu} / p_{\mathrm{T}}$, defined using the charmonium decay length $L_{x y}$ measured in the transverse plane. The fit model includes $J / \psi$ and $\psi(2 \mathrm{~S})$ contributions, as well as background components, and the use of $\tau_{\mu \mu}$ in the fits allows to separate charmonia yields from two types of production mechanisms:

- prompt charmonia - produced directly in the hard scattering of partons from the colliding nuclei and coming from feed-down from higher mass states;

- non-prompt charmonia - produced in decays of $B$-hadrons which occur outside of the QGP due to the relatively long $B$-hadron lifetimes.

The measurements in $p p$ and $p+\mathrm{Pb}$ collisions are combined to obtain $R_{p \mathrm{~Pb}}$ which is shown in Figure 1 as a function of $p_{\mathrm{T}}$ for promptly and non-promptly produced $J / \psi$. Both the prompt and non-prompt $R_{p \mathrm{~Pb}}$ are consistent with unity over the $p_{\mathrm{T}}$ range from 8 to $40 \mathrm{GeV}$ and no significant dependence on $p_{\mathrm{T}}$ is observed.
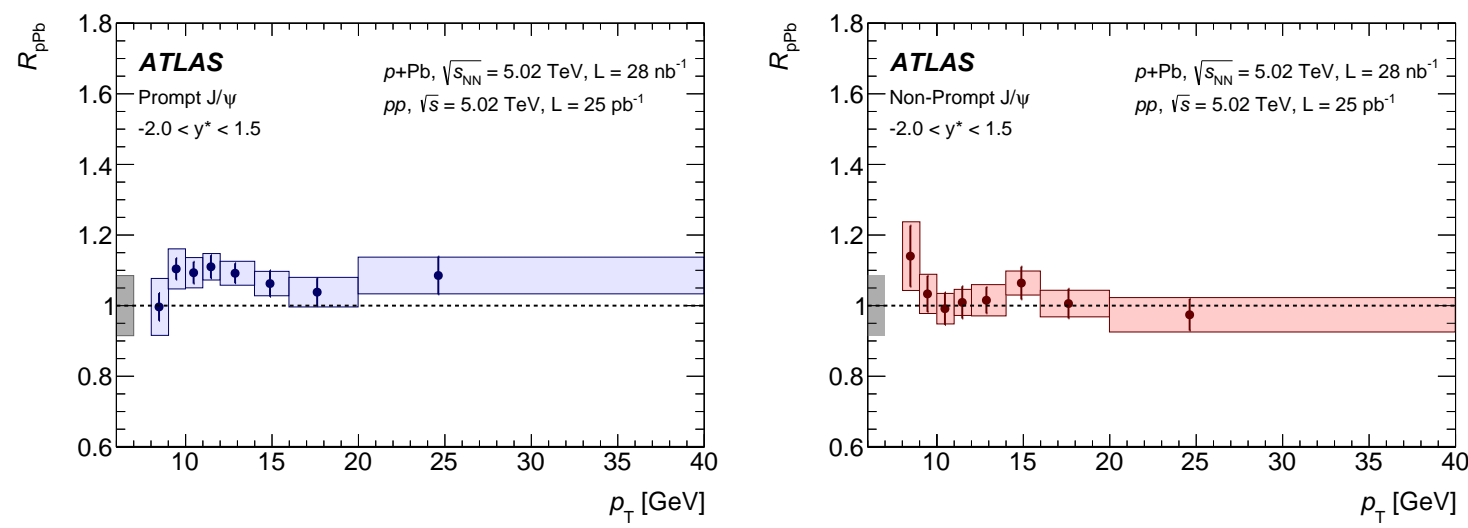

Figure 1: The nuclear modification factor, $R_{p \mathrm{~Pb}}$, as a function of transverse momentum $p_{\mathrm{T}}$ for prompt $J / \psi$ (left) and non-prompt $J / \psi$ (right). The horizontal position of each data point indicates the mean of the weighted $p_{\mathrm{T}}$ distribution. The vertical error bars correspond to the statistical uncertainties. The vertical sizes of coloured boxes around the data points represent the uncorrelated systematic uncertainties, and the horizontal sizes of coloured boxes represent the $p_{\mathrm{T}}$ bin sizes. The vertical sizes of the leftmost grey boxes around $R_{p \mathrm{~Pb}}=1$ represent the correlated systematic uncertainty [3].

The nuclear modification factor $R_{\mathrm{AA}}$ is obtained from a combination of measurements in $p p$ and $\mathrm{Pb}+\mathrm{Pb}$ collisions. Figure 2 presents $R_{\mathrm{AA}}$ for promptly and non-promptly produced $J / \psi$ as a function of centrality expressed as the number of nucleons participating in the collision $N_{\text {part }}$. For both production mechanisms, the suppression of production is strongly increasing with centrality. In the most peripheral collisions the $R_{\mathrm{AA}}$ is consistent with unity, while in the most central collisions it reaches a value around 0.2. Further measurements of the $R_{\mathrm{AA}}$ as a function of $p_{\mathrm{T}}$ and $y$ [4], which are not presented in this report, also reveal a modification of production which is very similar for promptly and non-promptly produced $J / \psi$ mesons.

Figure 3 presents the $R_{\mathrm{AA}}$ ratio between $\psi(2 \mathrm{~S})$ and $J / \psi$ mesons measured as a function of centrality, separately for promptly and non-promptly produced charmonia. For non-prompt 

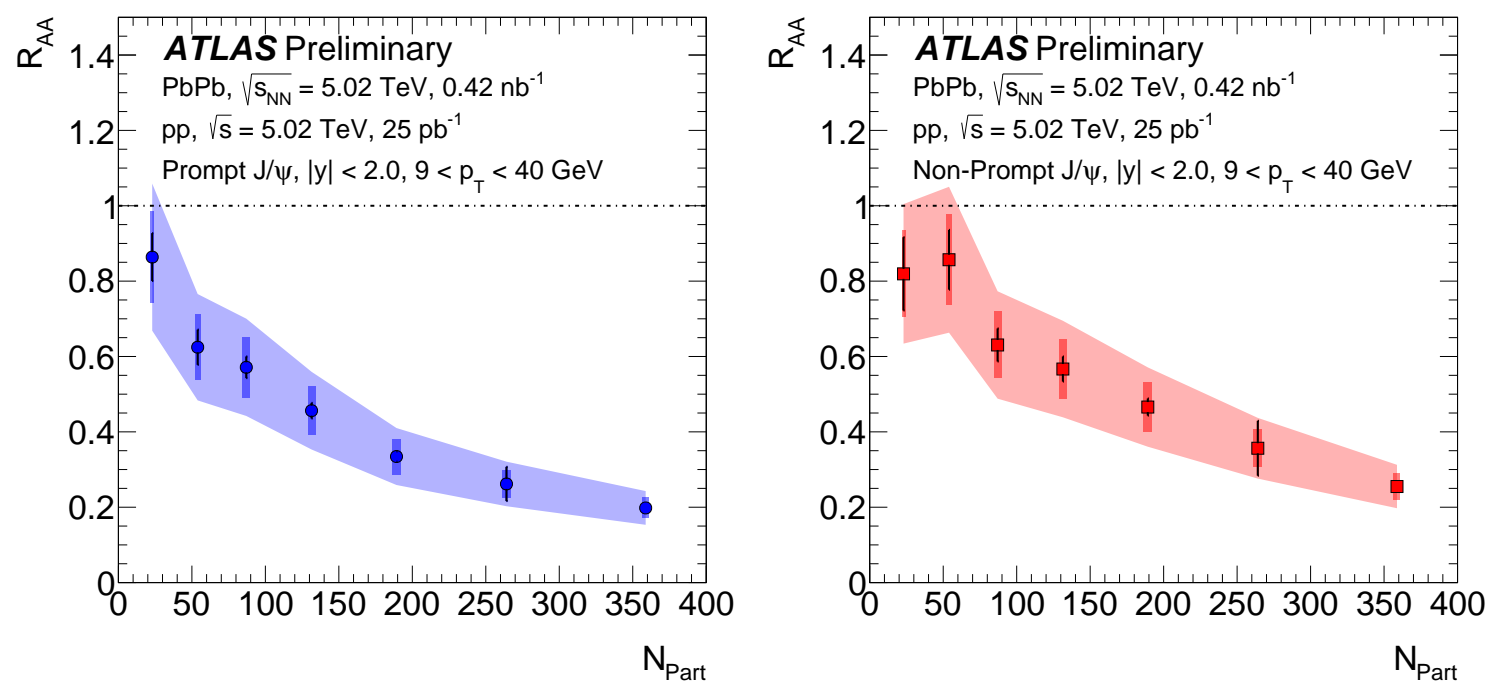

Figure 2: The nuclear modification factor as a function of centrality expressed as the number of participants $N_{\text {part }}$ for the prompt $J / \psi$ (left) and non-prompt $J / \psi$ (right) for $9<p_{\mathrm{T}}<40 \mathrm{GeV}$ and for rapidity $|y|<2$. The statistical uncertainty on each point is indicated by a narrow error bar which is smaller than the plotting symbol for most points. The wide error bar plotted with each point represents the point-to-point systematic uncertainty, while the error band connecting the points represents correlated scale uncertainties [4].

production the ratio is consistent with unity, while the prompt production of $\psi(2 \mathrm{~S})$ is found to be suppressed with respect to $J / \psi$ production.

\section{Bottomonia production in $p p$ and $p+\mathrm{Pb}$ collisions}

The production of bottomonia is studied in events collected with a di-muon trigger. The analysis strategy is similar to the one applied in the charmonia measurements, except for the $p_{\mathrm{T}}$ range: $p_{\mathrm{T}}<40 \mathrm{GeV}$.

Bottomonia yields are obtained from binned least squares fits in $m_{\mu \mu}$ which include contributions from the ground state $\Upsilon(1 S)$, two excited states $\Upsilon(2 S), \Upsilon(3 S)$ and a background component.

Figure 4 presents the nuclear modification factor, $R_{p \mathrm{~Pb}}$, measured for $\Upsilon(1 \mathrm{~S})$ as a function of $p_{\mathrm{T}}$ and centre-of-mass rapidity $y^{*}$. The nuclear modification factor is found to increase with $p_{\mathrm{T}}$ starting from a modest suppression at the lowest $p_{\mathrm{T}}$ and rising to unity above $15 \mathrm{GeV}$. As a function of rapidity, the $R_{p \mathrm{~Pb}}$ is measured to be approximately constant at the level of around 0.8 .

\section{Summary}

A summary of recent ATLAS measurements of quarkonia production in heavy-ion collisions is presented.

In $p+\mathrm{Pb}$ collisions, the CNM effects are observed only for the production of bottomonia, in particular in the suppression of $\Upsilon(1 \mathrm{~S})$ production at low $p_{\mathrm{T}}$, which suggests a nuclear modification of the parton distribution functions. No significant change of $J / \psi$ production is observed with respect to $p p$ collisions. 

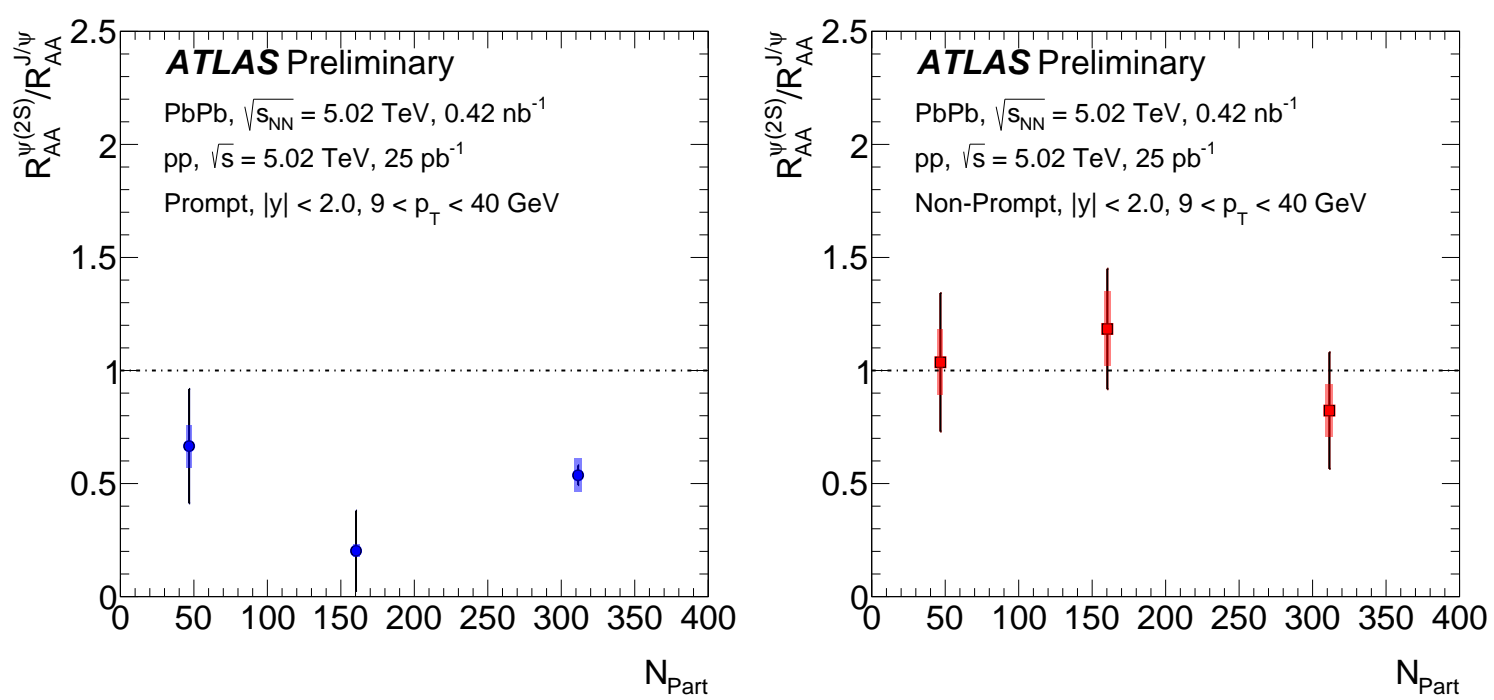

Figure 3: $R_{\mathrm{AA}}$ ratio for $\psi(2 \mathrm{~S})$ to $J / \psi$, as a function of centrality expressed as $N_{\text {part }}$, for prompt meson production (left) and non-prompt meson production (right). This ratio has the advantage that corrections are similar for the numerator and denominator, and thus systematic uncertainties are reduced. The narrow error bar represents the statistical uncertainties while the wider error bar represents the total systematic uncertainty [4].
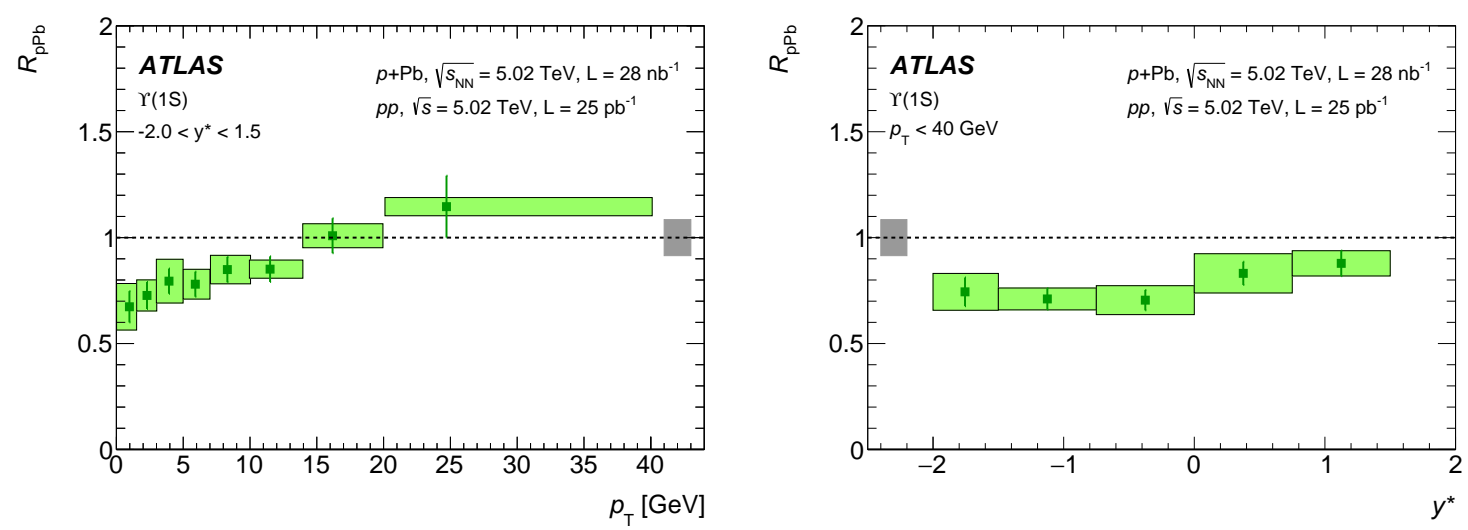

Figure 4: The nuclear modification factor, $R_{p \mathrm{~Pb}}$, as a function of transverse momentum $p_{\mathrm{T}}$ (left) and centreof-mass rapidity $y^{*}$ (right) for $\Upsilon(1 \mathrm{~S})$. The horizontal position of each data point indicates the mean of the weighted $p_{\mathrm{T}}$ or $y^{*}$ distribution. The vertical error bars correspond to the statistical uncertainties. The vertical sizes of coloured boxes around the data points represent the uncorrelated systematic uncertainties, and the horizontal sizes of coloured boxes represent the bin sizes. The vertical size of the rightmost (left) and leftmost (right) grey boxes around $R_{p \mathrm{~Pb}}=1$ represent the correlated systematic uncertainty [3]. 
In $\mathrm{Pb}+\mathrm{Pb}$ collisions, the QGP effects are observed very clearly, resulting in a strong suppression of charmonia production which increases with centrality. However, a surprisingly similar suppression pattern is observed for promptly and non-promptly produced charmonia, despite different production mechanisms. The ratio of nuclear modification factors measured for non-prompt $\psi(2 \mathrm{~S})$ and $J / \psi$ does not deviate from unity and is thus consistent with production in $B$-hadron decays outside of the medium. The same ratio measured for prompt charmonia is below unity which supports the hypothesis of a sequential melting of higher mass states.

This work was supported in part by the National Science Center grant UMO-2015/19/B/ST2/00906 and by PL-Grid infrastructure.

\section{References}

[1] T. Matsui, H. Satz, Phys. Lett. B 178 (416) 1986.

[2] ATLAS Collaboration, JINST 3 (2008) S08003.

[3] ATLAS Collaboration, arXiv:1709.03089 [nucl-ex].

[4] ATLAS Collaboration, ATLAS-CONF-2016-109, https://cds.cern.ch/record/2220771. 